Audiology

Neurotology
Audiol Neurotol 2011;16:323-335

DOI: $\underline{10.1159 / 000322504}$
Received: July 29, 2010

Accepted after revision: October 29, 2010

Published online: December 19, 2010

\title{
Distribution of Dexamethasone and Preservation of Inner Ear Function following Intratympanic Delivery of a Gel-Based Formulation
}

\author{
Alec N. Salt ${ }^{a}$ Jared Hartsock ${ }^{a}$ Stefan Plontke ${ }^{c}$ Carl LeBel $^{b} \quad$ Fabrice Piu ${ }^{b}$ \\ a Department of Otolaryngology, Washington University School of Medicine, St. Louis, Mo., and \\ b Otonomy Inc., San Diego, Calif., USA; ' Department of Otorhinolaryngology, Head and Neck Surgery, \\ University of Halle, Halle, Germany
}

\section{Key Words}

Cochlea $\cdot$ Perilymph $\cdot$ Round window $\cdot$ Intratympanic drug delivery $\cdot$ Dexamethasone

\begin{abstract}
Intratympanic (IT) delivery of drugs to the ear is increasingly used for both clinical and research purposes. One limitation of IT delivery is that drugs are rapidly lost from the middle ear by a number of processes, so that prolonged delivery of drug is technically difficult. In the present study, the delivery characteristics of a poloxamer hydrogel formulation containing dexamethasone (dex) were evaluated. The gel is liquid at room temperature, allowing IT injection, but transitions to a gel at body temperature, providing a prolonged residence time in the middle ear. A $50-\mu$ l volume of control or dex-containing gel (dex-gel) was injected through the tympanic membrane of guinea pigs. Cochlear function was assessed with cochlear action potential and acoustic emission thresholds measured immediately, 6 or $24 \mathrm{~h}$ after IT gel injection. After 6- or 24-hour treatment with dex-gel, perilymph drug gradients along the cochlea were assessed by taking samples sequentially from the apex, and endolymph was sampled from the basal turn. Control gel injections caused small changes in sound field calibrations and functional measures for low-frequency stimuli, consistent with
\end{abstract}

\section{KARGER}

Fax +4161306 1234

E-Mail karger@karger.ch

www.karger.com (c) 2010 S. Karger AG, Basel

Accessible online at: www.karger.com/aud an induced conductive loss. Within $24 \mathrm{~h}$, responses returned to normal. Twenty-four hours after dex-gel injection, lowfrequency changes remained as the dex-gel was retained better in the middle ear, but there was no indication of highfrequency loss. While perilymph sample data showed that dex gradients were substantially lower than after single injections of dex solution, quantitative analysis of this result suggests that some dex may have entered the perilymph through the thin bone in the apical region of the cochlea. Endolymph levels of dex remained lower than those in the perilymph. This study confirms that a poloxamer hydrogelbased dex formulation provides an effective method for a prolonged delivery, providing a more uniform distribution of drug in the inner ear.

Copyright $\odot 2010$ S. Karger AG, Basel

\section{Introduction}

Intratympanic (IT) steroids have become widely used to treat sudden sensorineural hearing loss, Ménière's disease and autoimmune ear disorders [Alles et al., 2006; Hu and Parnes, 2009]. Although some groups have applied drugs in a sustained manner using microcatheters and microwicks [Kopke et al., 2001; Herr and Marzo, 2005; Van Wijck et al., 2007; Plontke et al., 2009], the vast ma- 
jority have applied them as single or as repeated IT injections (up to 8 injections over periods of up to 2 weeks).

Intratympanically applied drugs are thought to enter the fluids of the inner ear primarily by crossing the round window (RW) membrane. Calculations show that a major factor controlling both the amount of drug entering the ear and the distribution of drug along the length of the ear is the duration the drug remains in the middle ear space [Salt et al., 2008]. Single, 'one-shot' applications or applications of aqueous solutions for few hours' duration result in steep drug gradients for the applied substance along the length of the cochlea [Plontke et al., 2008a] and rapidly declining concentration in the basal turn of the cochlea as the drug subsequently becomes distributed throughout the ear [Plontke and Salt, 2006; Salt and Plontke, 2009]. One-shot therapies are appropriate for drugs such as gentamicin, a treatment for Ménière's disease, because a critical aspect of this therapy is to minimize drug spread along the cochlea and hence minimize hearing loss as a potential adverse effect of the treatment. On the other hand, for treatments in which it is desired to deliver a more uniform drug level throughout the inner ear, a prolonged application of drug is preferable. This gives the drug sufficient time to diffuse throughout the fluid spaces of the ear, allowing a more even distribution to be achieved. Maintaining a prolonged drug application to the ear, however, is technically challenging. Injected drug solutions can be lost either down the eustachian tube during swallowing or through the middle ear mucosa to the vascular system. The middle ear mucosa is ciliated [Hentzer, 1984], which aids the removal of fluids from the compartment. Even if the injected fluid is retained in the middle ear, the loss of drug from the fluid over time can be remarkably high. Measurement of a marker ion (trimethylphenylammonium) in the RW niche of guinea pigs after the bulla was filled with solution showed that the ion concentration fell to an average of $55 \%$ of the injected concentration in just $30 \mathrm{~min}$ [Mikulec et al., 2009]. In a computer simulation of a one-shot IT methylprednisolone application in humans [Bird et al., 2007], the inclusion of an elimination half-time of $27 \mathrm{~min}$ from the middle ear provided the closest fit to the measured data [Plontke et al., 2008b].

In order to provide a prolonged delivery of drug to the cochlea, it is therefore necessary to control the major 2 forms of drug loss from the middle ear, specifically drug volume loss down the eustachian tube and drug clearance from the fluid by the middle ear mucosa. As the poloxamer hydrogel formulation of dexamethasone (dex) forms a gel (dex-gel) at body temperature, it minimizes volume loss via the eustachian tube. In addition, by utilizing this slowly dissolving form of dex, a sustained drug concentration is maintained in the middle ear even with ongoing drug clearance by the middle ear mucosa. Preliminary studies have demonstrated that following a single IT administration of the dex-gel, significant drug levels in the perilymph were maintained for at least 10 days [Wang et al., 2009]. The present study has evaluated the pharmacokinetic and functional effects of this delivery system.

\section{Methods}

This study utilized 33 pigmented NIH strain guinea pigs from our own colony, of both sexes and weighing 450-600 g. Animals were either assigned to a control group or to 1 of 4 groups injected intratympanically with a gel-based formulation either containing dex or without dex as detailed below. In each animal, the auditory bulla was later exposed by a ventral approach, the bulla was opened and a ball electrode was placed on the apex of the cochlea in order to measure cochlear evoked potentials. Acoustic emission $\left(2 f_{1}-f_{2}\right)$ thresholds were also established from a microphone system in the external ear canal. For those animals treated with the dex-containing gel formulation, sequential samples of perilymph were taken from the cochlear apex in order to determine the gradient of dex along the cochlea. A single sample of endolymph was also taken. These animal studies were conducted in accordance with the policies and recommendations of the United States Department of Agriculture, the National Institutes of Health guidelines for the handling and use of laboratory animals, and received approval from the Institutional Animal Care and Use Committee of Washington University under protocol 20070147.

\section{Drug Formulation}

The gel-based drug formulations used in this study were identical to those described previously [Wang et al., 2009]. A $20 \%$ (w/w) stock solution of poloxamer 407 gel (Spectrum Chemical MFG Corp., Gardena, Calif., USA) was prepared by slowly adding it to cold $10 \mathrm{~mm}$ phosphate-buffered saline at $\mathrm{pH}$ 7.4. Additional buffer solution was added to obtain a $17 \% \mathrm{w} / \mathrm{w}$ concentration of poloxamer 407 gel. This solution is liquid when refrigerated or at room temperature but solidifies at body temperature. The gel was tinted blue with Evans blue dye (50 ppm) and was sterilized by filtration. Using aseptic techniques, sterilized micronized dex (pure dex in a crystalline, powder form; Pfizer Inc., Kalamazoo, Mich., USA) was suspended with an appropriate amount of sterile poloxamer 407 solution to obtain a $4.5 \%$ solution. Samples of the formulation were stored under refrigeration and resuspended immediately before administration.

Animal Groups and Anesthesia

The groups used in this study consisted of:

(1) non-injected controls $(n=9)$ : the ear was exposed and recordings were made without any prior injection into the tympanic cavity; 
(2) 'gel-no-dex', immediate recording $(n=9)$ : gel containing no dex was injected intratympanically followed by immediate exposure of the ear and physiologic recordings;

(3) 'gel-no-dex', 24-hour recording $(n=4)$ : gel containing no dex was injected intratympanically and the animal was allowed to recover for $24 \mathrm{~h}$ before physiologic recordings were made;

(4) 'dex-gel', 6-hour recording $(n=5)$ : gel-containing dex was injected intratympanically and the animal was maintained under anesthesia for $6 \mathrm{~h}$ before the bulla was opened, physiologic recordings made and cochlear fluids sampled;

(5) 'dex-gel', 24-hour recording $(n=6)$ : gel-containing dex was injected intratympanically and the animal was allowed to recover for $24 \mathrm{~h}$ before physiologic recordings were made and cochlear fluids sampled.

For animals undergoing recovery surgery (groups 2 and 5) anesthesia induction used $50 \mathrm{mg} / \mathrm{kg}$ ketamine and $5 \mathrm{mg} / \mathrm{kg}$ xylazine, followed by maintenance on $0.7-1.1 \%$ isoflurane in oxygen. However, as xylazine is a potent diuretic and can potentially influence cochlear fluid status, animals undergoing nonrecovery surgery were initially anesthetized with $100 \mathrm{mg} / \mathrm{kg}$ Inactin (sodium thiobutabarbital) and maintained on $0.7-1.1 \%$ isoflurane in oxygen. In these animals, a tracheal cannula was placed and they were mechanically ventilated. Heart rate and vascular $\mathrm{pO}_{2}$ were monitored with a pulse oximeter (Surgivet, Waukesha, Wisc., USA) and end-tidal $\mathrm{CO}_{2}$ was maintained near $38 \mathrm{~mm} \mathrm{Hg}$ (5\%). Rectal temperature was maintained at $38^{\circ} \mathrm{C}$ with a DC-powered, thermistor-controlled heating blanket. Prior to physiologic recordings, pancuronium bromide $(2 \mathrm{mg} / \mathrm{ml})$ was given intravenously as a muscle relaxant to reduce myogenic artifacts and to eliminate middle ear muscle contractions.

\section{Intratympanic Injection Procedure}

The head of the anesthetized animal was held in a head holder, oriented with the nose pointing vertically upwards. The nose was inserted into a nose cone delivering anesthetic gas. The head was held in position against a foam pad on the left side of the head using a Velcro strap. A custom-made speculum, mounted on a manipulator, was positioned in the right external ear canal, allowing the tympanic membrane to be visualized. Perforations of the tympanic membrane were performed with a sterile needle of 28 gauge $\times 1$ inch, bent $90^{\circ}$ near the hub, so the syringe on which it was mounted did not obscure the view down the ear canal. The syringe and needle were mounted on a manipulator. A small perforation of the tympanic membrane was first made anterior to the malleus to provide a vent hole. The speculum was readjusted and a second perforation of the tympanic membrane was made as far posterior and dorsal as possible. IT injection of solutions was performed with a sterile Hamilton $50-\mu l$ syringe driven by a motorized WPI Ultrapump system. A blunt, sterile 28-gauge needle, bent $90^{\circ}$ near the hub, was mounted on the syringe and was inserted through the tympanic membrane perforation with the aid of a micromanipulator. When in place, $50 \mu$ l of gel-based suspension was injected into the RW niche area at a rate of $2 \mu \mathrm{l} / \mathrm{s}$, driven by the motorized system. It has been determined empirically that $50 \mu \mathrm{l}$ were sufficient to fill the RW niche and lateral bulla while limiting contact with anterior regions of the bulla, including the apex of the cochlea [Wang et al., 2009]. The animal remained in position for $5 \mathrm{~min}$ for the gel to solidify before being removed from the holder.

Intratympanic Gel-Based

Dexamethasone

\section{Measurements of Cochlear Function}

Animals were mounted in a head holder using hollow ear bars, allowing sounds to be delivered in a closed system. The right ear canal was transected to facilitate ear bar placement. Sound stimuli were generated and data were collected using Tucker Davis System 3 hardware under the control of custom-written software running on a PC. Sound stimuli were delivered and sound levels monitored with an Etymotic ER10C emissions system with probe tubes terminating close to the tip of the ear bar.

In each animal, the sound fields for both speakers were calibrated in an automated procedure from $125 \mathrm{~Hz}$ to $22.6 \mathrm{kHz}$ in $1 / 4$ octave steps (31 frequencies). At each frequency, the attenuation required to generate a sound level in the external canal within \pm 1 $\mathrm{dB}$ of $70 \mathrm{~dB}$ SPL was established. Calibration tables were calculated for each frequency in each animal that allowed all subsequent stimuli to be delivered on a dB SPL basis.

Cochlear sensitivity was assessed using an automated, objective algorithm to determine cochlear action potential (CAP) thresholds. The CAP response was recorded from an $\mathrm{Ag} / \mathrm{AgCl}$ ball electrode placed on the cochlear apex, measured relative to a platinum needle electrode at the vertex using a Tucker Davis HB4 amplifier. An amplifier gain of $\times 1000$ was used, with high-pass filtering of the signal at $200 \mathrm{~Hz}$. The apical recording site was chosen, rather than the commonly used RW recording site, to avoid variations in electrical pickup caused by the drug and gelfilled RW niche. Stimuli were 12-ms duration tone bursts with 0.5 -second cosine-gated onset. Responses to 10 noninverted and 10 inverted stimuli were averaged to obtain the CAP response with minimum contamination by cochlear microphonic components. Thresholds were established in a fully automated procedure using a $10-\mu \mathrm{V}$ response criterion, combined with verification that measured peak levels without stimulation were less than $5 \mu \mathrm{V}$. Stimuli were increased in 5 - $\mathrm{dB}$ steps until a response exceeding the criterion was obtained, and then reduced in $5-\mathrm{dB}$ steps until the response was below the criterion. A threshold (in $\mathrm{dB}$ SPL) was established by linear interpolation between the above- and below-threshold response amplitudes. CAP thresholds were determined in $1 / 4$ octave steps between 1 and $22.6 \mathrm{kHz}$ (19 frequencies).

Acoustic emissions were measured from the microphone sealed into the external ear canal. Ten 4096-point, phase-locked buffers were time averaged and subjected to fast Fourier transform analysis to derive a response spectrum. Two spectra were averaged before peak measurements were made. Stimulus frequencies were optimized so that an exact number of cycles occurred in the collection buffer, and no window function was necessary for the fast Fourier transform. Stimulus $\mathrm{f}_{2}$ frequency was set to be 1.2 times $f_{1}$, with an amplitude of $10 \mathrm{~dB}$ below $f_{1}$. The threshold of the $2 f_{1}-f_{2}$ response was derived in a fully automated procedure in which a $2 f_{1}-f_{2}$ response of $10 \mathrm{~dB}$ greater than the adjacent noise floor and $10 \mathrm{~dB}$ greater than the same frequency location in the absence of primaries indicated a positive response. The same algorithm was used as for CAP thresholds (increasing $5 \mathrm{~dB}$ in the absence of the criterion $2 \mathrm{f}_{1}-\mathrm{f}_{2}$ response and decreasing $5 \mathrm{~dB}$ when the response was present). $2 \mathrm{f}_{1}-\mathrm{f}_{2}$ emission thresholds were plotted against the $\mathrm{f}_{2}$ frequency. Thresholds were determined for $f_{1}$ varied in $1 / 4$ octave steps from $500 \mathrm{~Hz}$ to $8 \mathrm{kHz}$ (17 frequencies).

In each animal, all functional measurements were performed prior to fluid sampling procedures, which are described below. 
Cochlear Fluid Sampling and Analysis

Perilymph samples were collected sequentially from the cochlear apex in a manner that allows the gradients along the scala tympani (ST) to be derived [Mynatt et al., 2006]. The rationale underlying the method is that when all fluid emerging is collected without any loss to the bulla, then the samples can be interpreted quantitatively in terms of the perilymph concentration prior to sampling. Fluid was collected without loss by constructing a cup made of silicone glue around the apex prior to perforation of the bone, as detailed previously [Mynatt et al., 2006]. In brief, the bone at the apex of the cochlea was cleared of mucosa and was washed twice with lactated Ringer's solution before being dried with tissue wicks. A thin layer of cyanoacrylate adhesive was applied to the dry bone and covered with a layer of a two-part silicone adhesive (WPI Kwik-Cast). Multiple layers were built around the periphery until a 'cup' shape was formed. Perforation of the bony otic capsule at the apex allowed fluid to emerge that was collected in hand-held glass capillary tubes, marked at $1 \mu \mathrm{l}$ in volume. Eight separate fluid samples were collected from each animal. Fluid sampling failed in 1 animal, when the apical bone cracked during fenestration. For the 80 perilymph samples in the study, the mean volume collected in each sample (determined by length measurement of the sample in the capillary tube) was 1.04 $\mu l$ and the average time to collect each sample was $37 \mathrm{~s}$. It has been shown that the initial sample contains fluid that originated from the apical turns of the ST and successive samples contain perilymph originating from more basal regions of the cochlea, followed by cerebrospinal fluid that has passed through the ST [Mynatt et al., 2006]. In each animal, a single endolymph sample was taken from the basal turn. A double-barreled pipette, beveled to a diameter of 10-15 $\mu \mathrm{m}$, was inserted into endolymph through a fenestration in the lateral wall. One barrel was filled with 500 $\mathrm{mM} \mathrm{KCl}$ and was used to record endocochlear potential to verify tip placement within the endolymphatic space. The second pipette barrel contained mineral oil colored with Sudan black dye. When the tip was verified to be in the endolymphatic space, suction was applied to the sample barrel until an endolymph sample was aspirated. Each cochlear fluid sample was expelled into $50 \mu \mathrm{l}$ of a 50:50 methanol:water mixture. Dex concentrations were measured using high-pressure liquid chromatography combined with mass spectrometry detection, as detailed by Wang et al. [2009]. The limit of detection of the method was $1.0 \mathrm{ng} / \mathrm{ml}$.

Fluid sample data were interpreted with the aid of a simulation program (available at http://oto.wustl/edu/cochlea/) that was modified to include algorithms for drug entry into the perilymph through the bony capsule [Mikulec et al., 2009] and the fluid movements associated with sequential perilymph sampling from the apex [Mynatt et al., 2006]. Simulation of the sequential sampling procedure was based on the 8 specific sample volumes and times obtained in each experiment, or sample volumes and times averaged for the experimental groups as appropriate.

\section{Statistical Analysis}

Two-way analysis of variance (Sigmastat software) was used to establish statistical significance between groups. The Holm-Sidek test was used to establish significant differences from the control, non-injected group, with a probability level of $<0.05$ considered significant.

\section{Results}

Sound levels measured in the external canal to some stimulus frequencies were altered by gel injections into the middle ear, as shown in figure 1 . The curves shown represent the sound level (in $\mathrm{dB}$ SPL) for no attenuation, derived from the attenuation required to generate $70 \mathrm{~dB}$ SPL in the external canal. Immediately after gel-based medium without dex (gel-no-dex) was injected, sound levels to stimuli around $500 \mathrm{~Hz}$ were increased significantly and were decreased to stimuli below $250 \mathrm{~Hz}$. This is a likely result of a decreased mobility of the middle ear ossicles caused by the physical presence of the gel. After $24 \mathrm{~h}$ with gel-no-dex, sound field calibrations returned to normal. With dex-gel, sound levels measured for frequencies around $500 \mathrm{~Hz}$ were elevated both at 6 and $24 \mathrm{~h}$. The observation that low frequencies remained affected by dex-gel at $24 \mathrm{~h}$ but not by gel-no-dex is explained by the fact that dex-gel was retained better in the middle ear than was gel-no-dex. For the gel-no-dex the middle ear mucosa was often reddened by vasodilation, a reaction that was apparently suppressed by the presence of dex. The difference between the rates of gel clearance is thought to result from the different status of the middle ear mucosa. It is also possible that a viscosity difference between the dex-gel and the gel-no-dex could contribute to the observed differences.

Cochlear sensitivity changes caused by gel injections, as determined by CAP thresholds, are summarized in figure 2. Action potential thresholds were temporarily elevated by up to $10 \mathrm{~dB}$ across the frequency range by the gel-no-dex, which resolved by $24 \mathrm{~h}$. At 6 and $24 \mathrm{~h}$ after dex-gel, action potential threshold changes were minor, though significant for some lower frequencies. The lowfrequency sensitivity changes were small, but some persisted for $24 \mathrm{~h}$ or longer. There was no indication of any high-frequency hearing loss, corresponding to the regions closest to the RW and therefore expected to be exposed to the highest drug level. Generally similar results were observed with measurements of acoustic emissions as shown in figure 3. Immediately after gel-no-dex injection, emission thresholds to low frequencies were elevated, which partially resolved at $24 \mathrm{~h}$. Similarly, with dexgel, low-frequency thresholds were elevated at $6 \mathrm{~h}$, which partially resolved at $24 \mathrm{~h}$. Emissions at the higher frequencies tested were unaffected in all groups.

Dex levels measured in 8 sequential samples of perilymph taken from the cochlear apex and in endolymph are shown in figure 4 . At both 6 and $24 \mathrm{~h}$, a generally similar mean curve was obtained for the perilymph sam- 


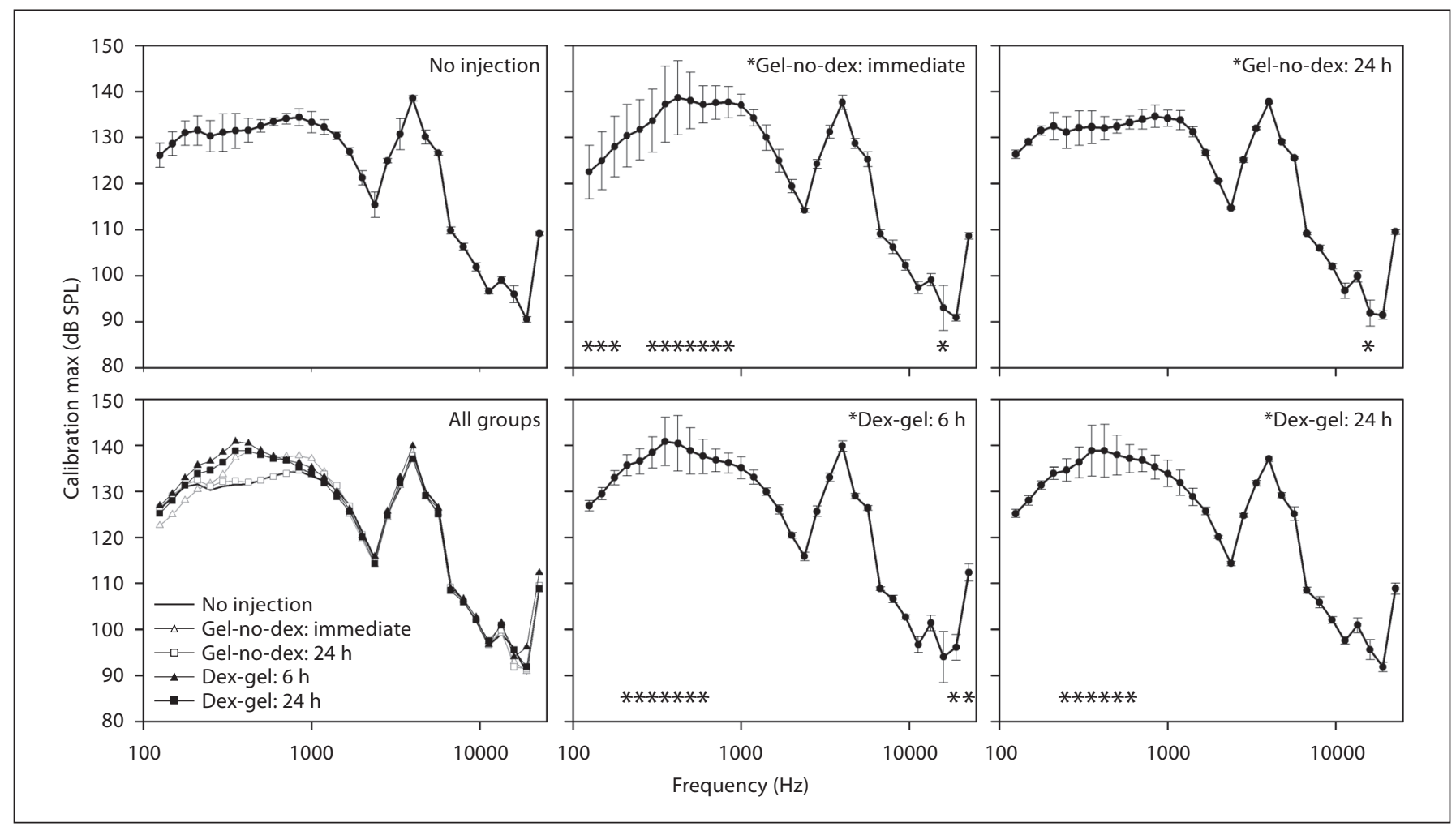

Fig. 1. Sound field calibrations (mean \pm SD) for the 5 experimental groups and the means for all groups overlaid (lower left panel). Values are the inferred maximum sound output with no attenuation, based on the amount of attenuation required to generate 70 $\mathrm{dB}$ SPL in the external canal. Groups that differ from the unin-

ples, although the concentrations were significantly higher at $24 \mathrm{~h}$ (mean of all apical samples $0.59 \mu \mathrm{g} / \mathrm{ml}$ ) compared to $6 \mathrm{~h}$ (mean of all apical samples $0.34 \mu \mathrm{g} / \mathrm{ml}$ ). In both groups, sample 1, containing perilymph that originated from the apical turns, was typically of higher concentration than sample 2, with concentrations then progressively increasing up to sample 4 , which originated from the basal cochlear turn. The shape of this curve (ignoring the absolute level differences that depend on the applied drug concentration and delivery protocol) differs substantially from samples taken in a similar manner after dex sodium phosphate solution was irrigated across the RW for 2-3 h. In this latter condition, the first sample was of very low concentration, increasing progressively to the 4th sample [Plontke et al., 2008a]. In contrast, when substances were applied by completely filling the bulla with drug solution, the first sample was found to be of very high concentration relative to the other samples, due to the substance entering the perilymph through the thin jected controls are indicated with an asterisk by the title. Individual frequencies that differ significantly from the uninjected controls are indicated by an asterisk below the data symbol. Gel in the middle ear primarily influenced frequencies around 500 $\mathrm{Hz}$.

bony capsule in the apical region of guinea pigs [Mikulec et al., 2009]. In the present study, we therefore needed to consider the possibility that drug could have entered the perilymph through the thin bone at the apex, in addition to that entering through the RW membrane. Visual inspection of the location of the blue-tinted dex-gel when the bulla was opened showed that the blue gel was primarily located around the ossicles and RW niche, but in some animals (3/11), the blue gel was present in the bulla near the cochlear apex.

As with our previous studies, we have used computer simulation of dex movements within the cochlear fluids to help interpret the measured sample data. In order to account for samples 1 and 4 being of higher concentration than samples 2 and 3, the simulation required both dex entry into the basal turn of the ST through the RW membrane and a dex entry into the apical turn of the cochlea corresponding to entry through the bone. In the simulation of the experiments shown in figure 5 , the rate of dex 


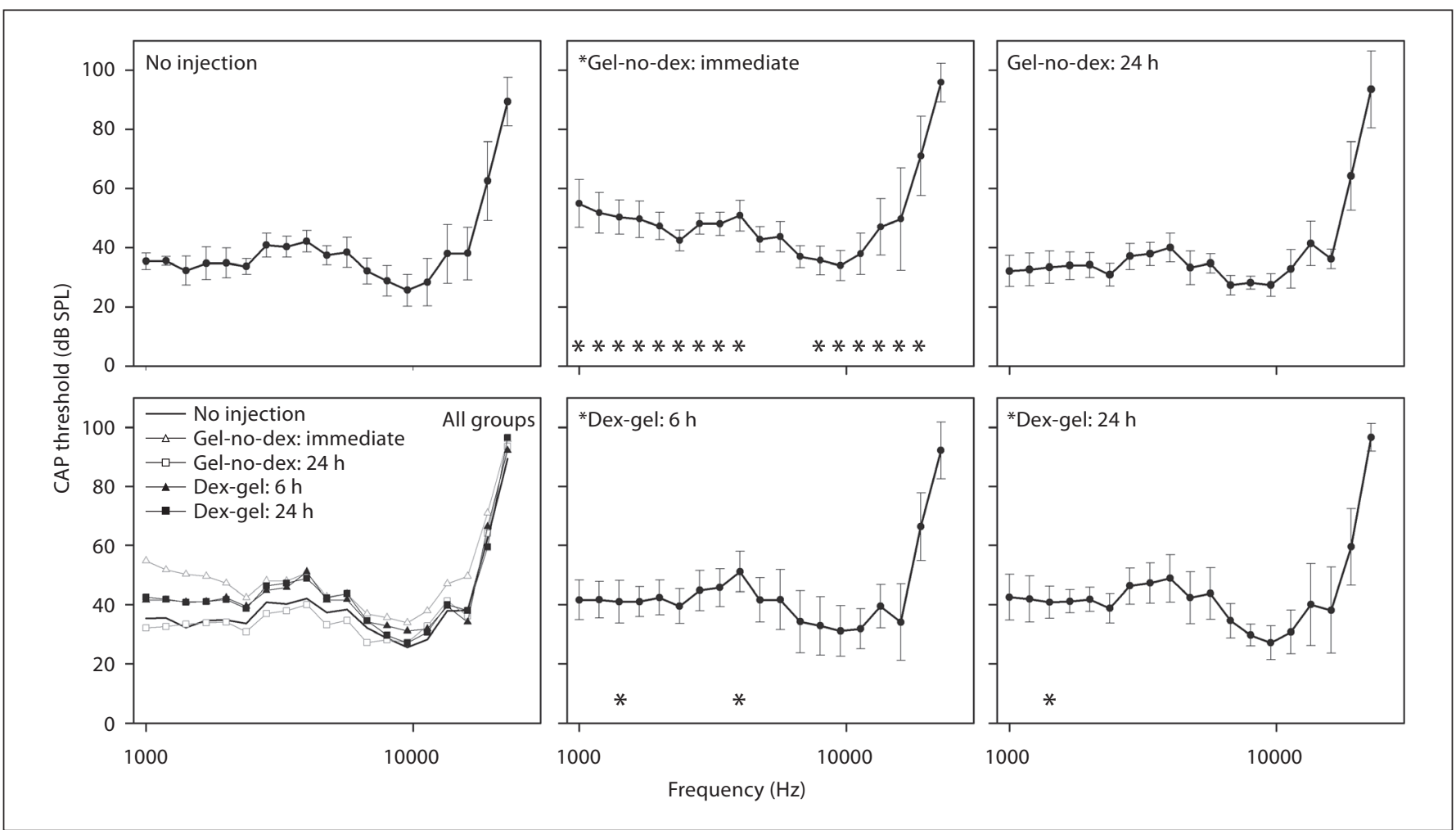

Fig. 2. Cochlear sensitivity (mean $\pm \mathrm{SD}$ ) determined by CAP thresholds for the 5 experimental groups and the means of all 5 groups overlaid (lower left). Groups that were significantly different from the no injection controls are indicated by an asterisk in the title. Action potential thresholds at individual frequencies that differed from the controls are indicated by an asterisk below

entry into the apical 2-mm segment of the ST and scala vestibuli was varied to account for the concentration detected in sample 1. The permeability of the RW membrane was simultaneously adjusted so that the calculated average of samples 4,5 and 6 was the same as that measured experimentally. The shape of the curve (i.e. the magnitude of the 'dip' seen in sample 2) was very sensitive to the rate of drug elimination from the ST, as shown by the curves for different elimination rates in figure 5. By fitting the model for a range of elimination rates, it was possible to establish the elimination rate that best accounted for the data, as shown in the lower panels of figure 5. The elimination rate that best fit the sample data obtained at $6 \mathrm{~h}$ after application was $85 \mathrm{~min}$ and the rate which best fit the 24-hour data was $155 \mathrm{~min}$. Details of the model parameters used to fit the 6- and 24-hour data are summarized in table 1 . The parameters providing the best fit to the 2 data sets are seen to differ from each other. This could either reflect variations between animals the symbol. Thresholds were elevated across most of the frequency range immediately after gel-no-dex injection, but this resolved at $24 \mathrm{~h}$. Only sporadic frequencies were elevated at 6 and $24 \mathrm{~h}$ after dex-gel. Thresholds to high stimulus frequencies were unaffected by dex-gel.

used in each experimental group or could indicate that some pharmacokinetic properties are varying with time. The simulations at present assume that pharmacokinetic processes do not change during the period of the simulation. For example, it is quite possible that RW membrane permeability increases with time of exposure to the nonphysiologic gel-fluid composition [Mikulec et al., 2008]. Similarly, the processes involved in drug elimination from the scalae may include drug movements to compartments, such as the modiolus, that decline with time as the compartment concentration increases. This would be seen as progressively increasing elimination half-time.

The calculated gradients of dex along the ST prior to sampling (those that gave rise to the sample values in fig. 5) are shown for both sampling times (dotted lines in fig. 6). As this figure shows concentration and does not consider the decreasing cross section of the ST towards the apex, it somewhat misrepresents the small amount of dex thought to be entering at the apex. From the simula- 


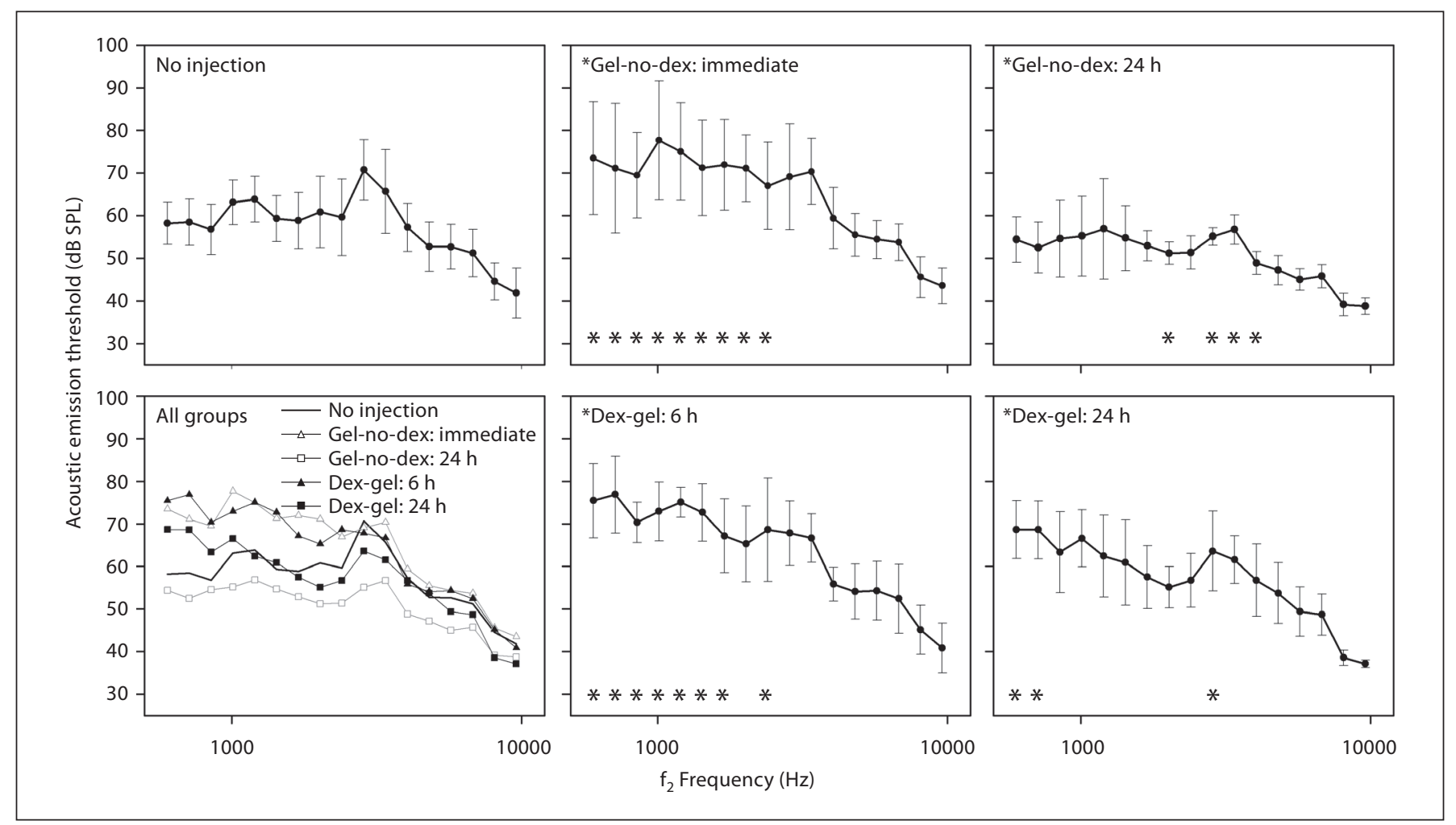

Fig. 3. Cochlear sensitivity (mean \pm SD) determined by acoustic emission thresholds for the 5 experimental groups and the means of all 5 groups overlaid (lower left panel). Groups that were significantly different from the no injection controls are indicated by an asterisk in the title. Emission thresholds at individual frequencies that differed from the controls are indicated by an aster- isk below the symbol. Thresholds to low-frequency stimuli were elevated after gel-no-dex injection, which largely resolved at $24 \mathrm{~h}$. Emissions to low-frequency stimuli were elevated at $6 \mathrm{~h}$ after dex-gel, but this largely resolved at $24 \mathrm{~h}$. Thresholds to the higher stimulus frequencies tested were unaffected by dex-gel.

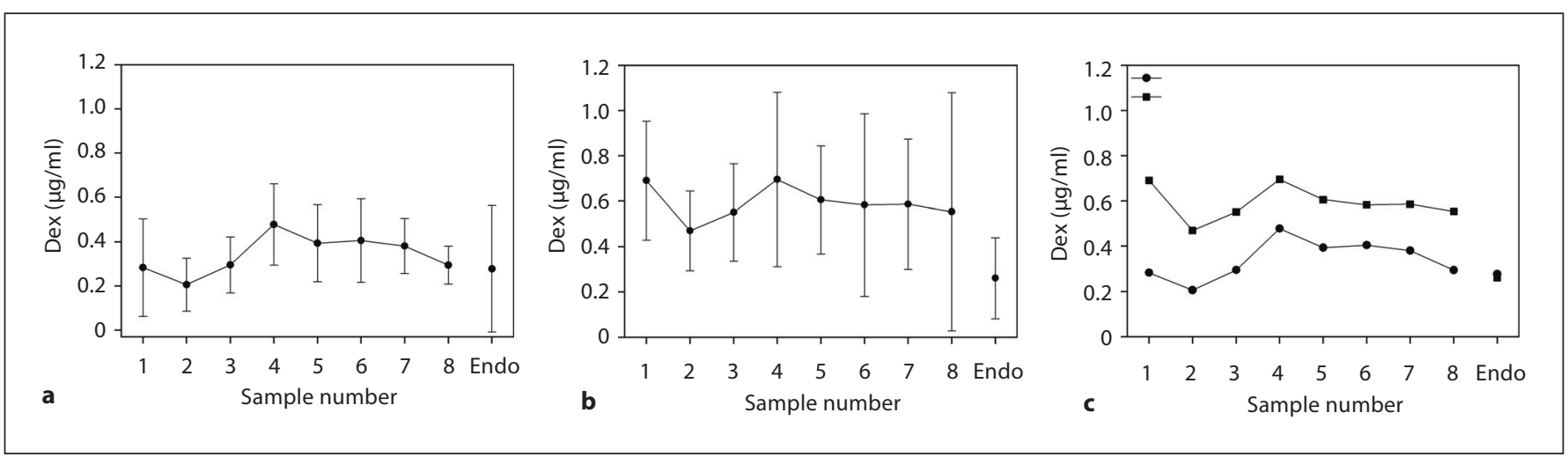

Fig. 4. Measured dex levels (mean $\pm \mathrm{SD}$ ) for 8 perilymph samples taken sequentially from the cochlear apex and for endolymph taken from the basal turn $6 \mathrm{~h}$ after dex-gel (a) and $24 \mathrm{~h}$ after dexgel (b). c The means of both groups are overlaid. In both groups, the first sample (originating in the apical turns) was higher than the second sample followed by a progressive increase to the 4 th sample. For both groups, the endolymph concentration was significantly lower than that of sample 4 (originating from the basal turn). 
Fig. 5. a, b Sample concentrations derived by calculation, fitted to the measured sample values for samples taken $6 \mathrm{~h}$ (a) and $24 \mathrm{~h}$ (b) after dex application. The measured sample curves, taken from figure 4 , are shown in gray. For each fitted curve apical entry through the bone was adjusted to account for sample 1 and RW membrane permeability was adjusted to account for the mean of samples 4, 5 and 6 . With this paradigm, sample 2 depends on the rate that dex is eliminated from the ST. Each of the curves is calculated with different ST elimination half-times as indicated in the legend. Curves with circles indicate those that best fit the sample data. c, d Variation of calculated sample 2 concentration as ST elimination rate was systematically varied. The lines indicate the elimination rates that best fit the concentration of sample 2, which were $85 \mathrm{~min}$ half-time for the 6-hour data and 155-min half-time for the 24-hour data.

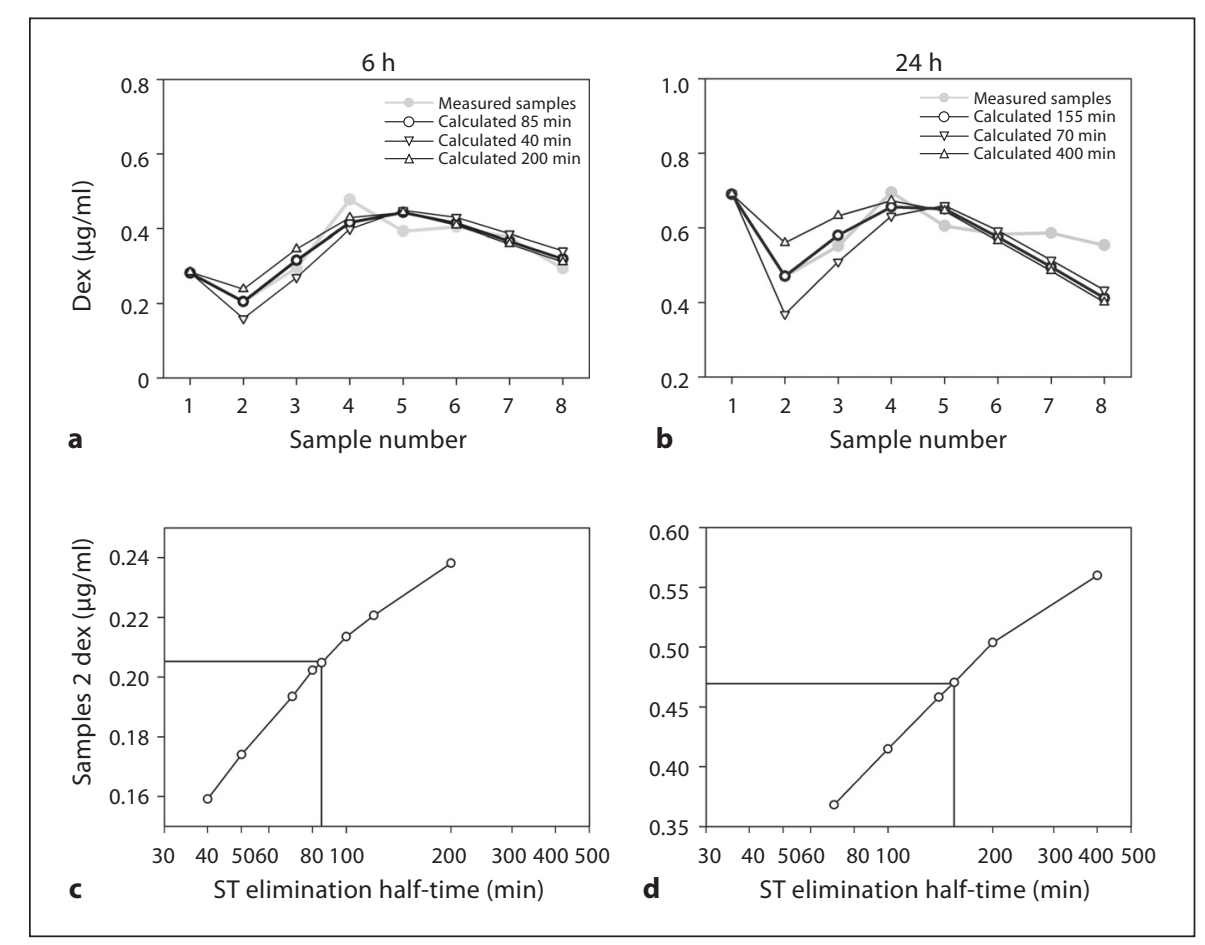

Table 1. Simulation parameters used to fit perilymph sample data

\begin{tabular}{|c|c|c|}
\hline & $\begin{array}{l}\text { Samples taken } \\
6 \mathrm{~h} \text { after application }\end{array}$ & $\begin{array}{l}\text { Samples taken } \\
24 \text { h after application }\end{array}$ \\
\hline RW membrane permeability & $1.19 \times 10^{-9} \mathrm{~m} / \mathrm{s}$ & $0.76 \times 10^{-9} \mathrm{~m} / \mathrm{s}$ \\
\hline Elimination half-time from ST & $85 \mathrm{~min}$ & $155 \mathrm{~min}$ \\
\hline Elimination half-time from SV and SM (set to $2 \times$ that in ST) & $170 \mathrm{~min}$ & $310 \mathrm{~min}$ \\
\hline Dex diffusion coefficient & $0.77 \times 10^{-9} \mathrm{~m}^{2} / \mathrm{s}$ & $0.77 \times 10^{-9} \mathrm{~m}^{2} / \mathrm{s}$ \\
\hline Perilymph flow rate & 0 & 0 \\
\hline Local interscala communication half-time (ST-SM, ST-SV, SM-SV) & $6 \mathrm{~min}$ & $6 \mathrm{~min}$ \\
\hline Access to $0.3 \mathrm{~mm}^{2}$ compartment parallel to $S T$ & $30 \%$ of free diffusion & $30 \%$ of free diffusion \\
\hline
\end{tabular}

Parameters shown in italics were held constant in all simulations. All other parameters were varied to fit the experimental data. Elimination half-time was assumed to be uniform along the length of each scala. SV = Scala vestibuli; $\mathrm{SM}=$ scala media.

tions, it was determined that just $9 \%$ of the dex entered the cochlea via the apical route (with $91 \%$ entering through the RW membrane) at $6 \mathrm{~h}$. The amount entering via the apical route increased to $18 \%$ at $24 \mathrm{~h}$, consistent with the increased distribution of gel around the bulla at this time. It is also notable that while the measured concentration of samples originating from the basal turn (samples 4, 5 and 6) was substantially higher at $24 \mathrm{~h}$ than at $6 \mathrm{~h}$ (fig. 4), sim- ulations of these data revealed that basal turn concentrations were similar for the 2 groups (fig. 6). This is because there is interaction between the basal turn ST perilymph and the adjacent cochlear compartments (scala media, scala vestibuli, modiolus, etc.) as the fluid moves along the ST to be collected at the apex [Mynatt et al., 2006]. In the case of the samples taken at $24 \mathrm{~h}$, regions higher up the cochlea were loaded with higher drug concentrations 
Fig. 6. Calculated concentration gradients along the ST at $6 \mathrm{~h}$ (gray) and $24 \mathrm{~h}$ (black) just prior to simulation of the sampling procedure. Dotted lines show gradients with dex entry through the RW membrane and through the bone at the apex, as need to fit the sample data. Solid lines show the calculated gradients with identical parameters but for RW entry only and dex entry at the apex excluded from the simulation.

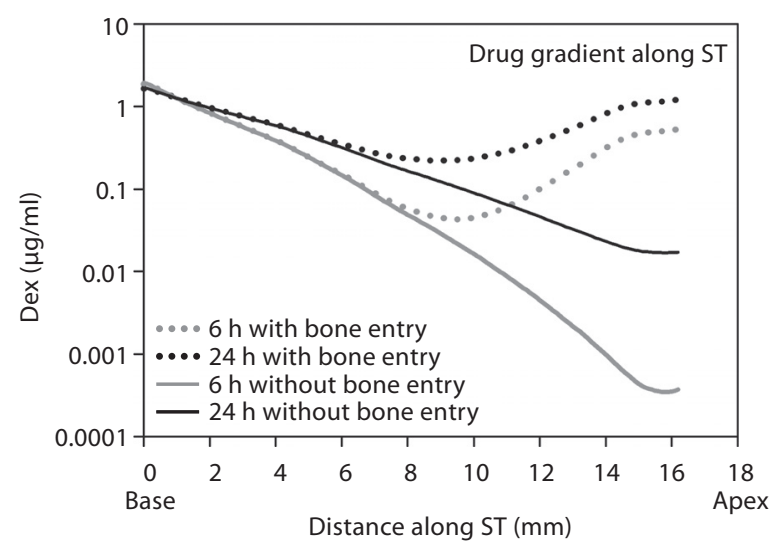

Distance along ST (mm)

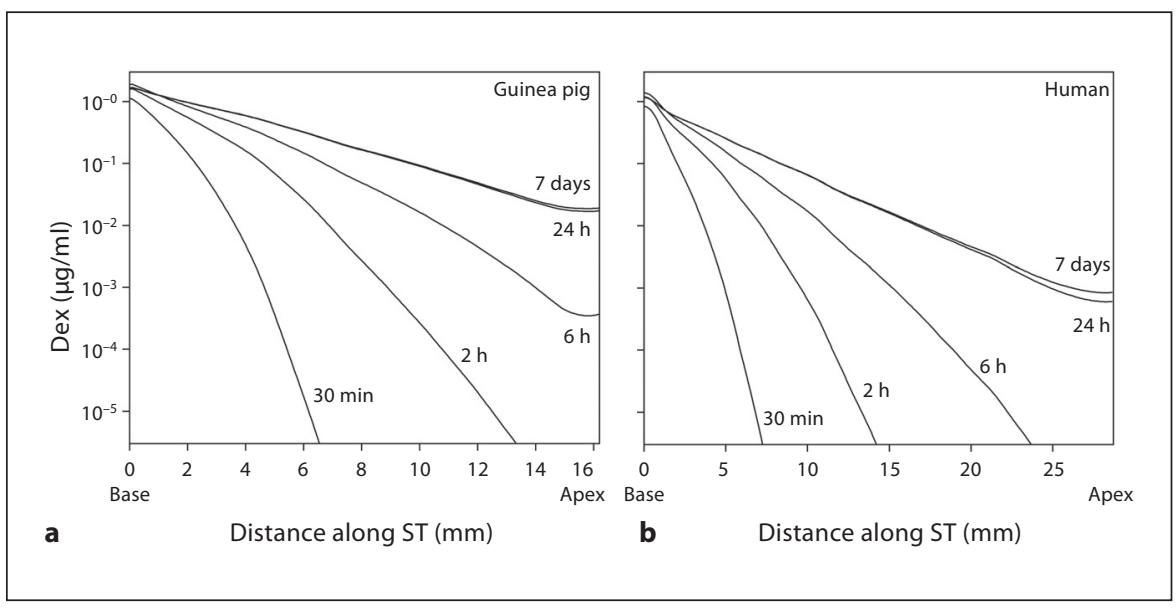

Fig. 7. Calculated gradients of dex along the ST for applications to the RW membrane of different durations for the guinea pig (a) and for the human (b). Entry into the perilymph through the bone at the apex of the guinea pig has been excluded from these calculations so that the distribution better represents the human situation. The duration of drug application has a major influence on the gradient along the length of the cochlea. In humans, the gradients will be larger than in the guinea pig because the cochlea is

(fig. 6, black dotted line), so the resulting sample concentrations were higher. As the simulations take into account such interactions during the sampling procedures, it underscores their value in the accurate quantitative interpretation of data. Figure 6 also shows for comparison the calculated longitudinal gradients of dex along the ST for entry only through the RW membrane, by excluding apical entry through the bone from the calculation. almost twice the length. The calculated curves for humans use scala dimensions and RW area appropriate for humans, but use pharmacokinetic parameters (RW membrane permeability, dex elimination rate, etc.) established in the guinea pig. Parameters derived from analysis of 6-hour data (table 1) were used for times up to and including $6 \mathrm{~h}$, and parameters from the analysis of 24hour data for times of $24 \mathrm{~h}$ or greater. figure 4 , averaged 0.28 and $0.26 \mu \mathrm{g} / \mathrm{ml}$ for the 6 - and 24 hour applications, respectively. The mean endolymph concentrations for both groups were less than those of the 4th sample of perilymph (which originates from the basal turn) which averaged 0.48 and $0.70 \mu \mathrm{g} / \mathrm{ml}$ for the 6and 24-hour applications, respectively. The difference was significant for the 24-hour condition but not for the 
6-hour condition. The endolymph concentrations were also substantially less than those derived from simulations for their adjacent basal turn ST locations (the 4-mm location in fig. 6), which were 0.8 and $0.94 \mu \mathrm{g} / \mathrm{ml}$, respectively. These endolymph concentrations represent 35 and $28 \%$ of the perilymph concentration at 6 and $24 \mathrm{~h}$, respectively.

\section{Discussion}

The present study shows that IT dex-gel offers a number of advantages over conventional injections of drug solutions into the middle ear. The measured perilymph drug levels both in this study and those reported by Wang et al. [2009] with similar methodology show less variability than prior studies with dex solutions [Parnes et al., 1999; Chandrasekhar et al., 2000; Hahn et al., 2006; Plontke et al., 2008a; Borden et al., 2010]. This is possibly the result of the longer residence time of the drug solution in the middle ear or the sustained drug level provided by the slowly dissolving micronized dex suspension. This sustained delivery will reduce, but not abolish, the longitudinal gradient for dex along the cochlea, as considered further below.

The poloxamer hydrogel formulation of dex was found to have a short-term influence on low-frequency sensitivity of the ear. Based on the significant changes in sound levels in the external ear canal determined in calibrations with control and dex-containing gels, the functional changes are thought to arise from the temporary, partial immobilization of the middle ear ossicles caused by the gel. There was no indication of high-frequency hearing loss or any indication of cochlear toxicity of dex at the concentrations achieved in the cochlea. These findings are consistent with prior studies showing no apparent indication of dex toxicity at the intracochlear concentrations achieved when applied to the RW niche [Wang et al., 2009]. It should also be noted that the individual sound field calibrations performed in the present study will produce slightly different results compared to similar physiologic measures using a fixed calibration performed in normal-hearing animals. For example, in our study the fact that significantly higher ear canal sound levels were found for gel-injected groups at $500 \mathrm{~Hz}$ results in the sound stimuli being delivered with more attenuation, so that action potential and distortion product otoacoustic emission threshold shifts at $500 \mathrm{~Hz}$ are greater than if a fixed calibration had been used. This approach makes the measurements more comparable with those obtained with free field stimuli. It also demonstrates, however, that threshold shifts measured using a closed sound system and a fixed calibration may underestimate low-frequency threshold shifts attributable to mechanical changes of the middle ear.

Interpretation of the pharmacokinetic measurements from this study required consideration of the routes by which dex entered the cochlea. In a previous study, we collected sequential samples of perilymph in an identical manner to those here following the irrigation of solution containing dex sodium phosphate across the RW membrane [Plontke et al., 2008a]. In that study (ignoring the absolute concentration differences due to different applied concentrations and delivery protocols), the initial sample taken from each animal had a low concentration of dex, with progressively increasing concentrations up to the 4 th or 5 th sample. The sequential sample data thus demonstrated the existence of sizeable gradients of drug along the cochlea, with concentration decreasing from the base to the apex by a factor that averaged $1.7 \times 10^{4}$. The sample concentrations measured at 6 or $24 \mathrm{~h}$ after IT injection of dex-gel in the present study showed a markedly different distribution. The initial sample in most animals (originating from the apical turns) was found to be of higher concentration than the second sample (which originated further towards the base of the cochlea). Previous studies have shown that when the bulla was completely filled with a drug-containing solution, the initial sample was found to be of high concentration due to drug entry into the apical perilymph through the thin bony capsule at the apex of the guinea pigs [Mikulec et al., 2009]. This interpretation was confirmed in that study by real-time measurements of marker entry into perilymph near the apex with ion-selective electrodes. In the present study, the low volume injected $(50 \mu \mathrm{l}) \mathrm{com}$ bined with the orientation of the animal during injection ensured that the applied drug was initially located in the posterior bulla and did not contact the apical regions. However, the data clearly show that this approach is insufficient to prevent entry at the apex for all guinea pigs. As the epithelium lining the bulla contains both secretory and ciliated cells [Hentzer, 1984], it is likely that there is mixing of free fluid in the bulla over a period of time, so that the mucosa overlying the cochlear apex could become contaminated with drug. In this respect, the raw data showing the drug distribution along the length of the guinea pig (fig. 4) therefore may not be an optimal model of the likely drug distribution in humans. In humans, the otic capsule is much thicker than in guinea pigs [Schuknecht, 1993], so drug entry through 
the bone is not likely to be significant. The use of simulations to fit the experimental data by inclusion of separate entry mechanisms through the RW membrane and through the bone at the apex allows the latter component to be quantified, and then removed from the calculation. This allows the distribution to be calculated for entry through the RW membrane only, excluding the apical entry that is specific to the guinea pig. Figure 7 shows the calculated gradients along the ST for the guinea pigs and the humans for different application durations assuming only entry through the RW membrane, and using parameters derived from dex-gel applications in guinea pigs. The importance of the duration of application in determining the magnitude of the drug gradient is clearly demonstrated. Substantial gradients persist even after prolonged applications due to the steady state established as the rate of diffusion along the scala balances the rate of elimination to blood. In contrast, however, the gradients are substantially larger following a 30-min application, as expected following a one-shot application. This demonstrates the substantial benefit provided by sustained delivery of the drug over a period of days or weeks. In these calculations, no specific difference is expected between whether the drug is delivered in solution or as a gel. A constant drug concentration in the RW niche over time is assumed. It has been reported that perilymph drug levels in guinea pigs may be maintained for over 10 days with this formulation [Wang et al., 2009], therefore providing a prolonged application with the drug distributed as widely in the cochlea as is currently possible.

The perilymph drug levels measured in the present study $(0.7 \mu \mathrm{g} / \mathrm{ml}$ after $24 \mathrm{~h})$, which were interpreted as being consistent with basal turn concentrations of approximately $1 \mu \mathrm{g} / \mathrm{ml}$ (fig. 6,7), are comparable to similar measurements reported by Wang et al. [2009], from samples taken $24 \mathrm{~h}$ after a similar application protocol, but using a different, basal turn perilymph sampling procedure. This concentration exceeds the concentration anticipated as necessary for therapeutic effects. In human tissues, dex is effective at concentrations ranging from 0.001 to $0.030 \mu \mathrm{g} / \mathrm{ml}$ [Czock et al., 2005]. Further, dex has been shown to modulate the activity of the epithelial sodium channel in the inner ear at concentrations of around $0.040 \mu \mathrm{g} / \mathrm{ml}$ [Pondugula et al., 2006; Kim et al., 2009], suggesting that the response of tissues of the ear to dex may be comparable to tissues outside the ear. These findings are consistent with therapeutic effects of dex being achieved when concentration exceeds $0.030-0.040 \mu \mathrm{g} /$ $\mathrm{ml}$. Based on the calculations in figure 7 , this concentra- tion would be exceeded for almost half of the cochlea in humans when applications maintain dex in the RW niche for $24 \mathrm{~h}$ or longer.

Other groups have reported the use of a variety of polymer and gel-based drug delivery systems. Arnold et al. [2005] used a polymer system to deliver beclomethasone with either slow or fast release kinetics into the RW niche. Endo et al. [2005] applied brain-derived neurotrophic factor to the RW niche in a collagen-based hydrogel. Tamura et al. [2005] delivered rhodamine to the RW niche with poly(lactic/glycolic acid) nanoparticles. Paulson et al. [2008] made a formulation of dex in a chitosan-glycerophosphate gel that was shown to provide a sustained application of dex to the perilymph of mice over a period of up to 5 days. James et al. [2008] compared intratympanic dex delivery for 4 types of polymer (gelatine, esterified hyaluronic acid, calcium alginate and modified hyaluronic acid and carboxymethylcellulose) and only found detectable levels of dex in perilymph with the hyaluronic acid/carboxymethylcellulose matrix. This demonstrates the importance of the choice of gel composition on drug entry into the cochlea. There have been some human studies in which dex combined with a low concentration of hyaluronic acid $(0.2 \mathrm{mg} / \mathrm{ml})$ was injected intratympanically in patients with sudden hearing loss or Ménière's disease [Gouveris et al., 2005; Selivanova et al., 2005]. In these studies, the hyaluronate was not intended to act as a gel to stabilize volume, but rather was intended to increase the rate of entry into perilymph by permeabilizing the RW membrane. More recently, a higher concentration of hyaluronate $(6 \mathrm{mg} / \mathrm{ml})$ has been used in combination with dex to provide a hydrogel for volume stabilization [Borden et al., 2010]. With this method, measurable dex levels in perilymph could be maintained for up to 3 days in guinea pigs. The use of sustained-release, gel-based formulations therefore seems to provide a good solution to optimize drug distribution in the cochlea with a single IT delivery procedure. In clinical practice, the single IT injection required by this approach is preferable to the use of implanted catheters and pumps to provide sustained delivery [Kopke et al., 2001; Plontke et al., 2009]. The results of the present study suggest that the dex-gel formulation we have used is worthy of clinical evaluation for the treatment of steroid-responsive inner ear disorders.

The endolymph samples in this study showed lower concentrations than perilymph at both time points measured. This suggests that this form of dex is not accumulated in endolymph during a prolonged application pro- 
cedure. This finding contrasts with the observation of higher endolymph concentrations than perilymph by Parnes et al. [1999], but this could be accounted for by experimental differences. The experiments by Parnes et al. used dex phosphate, which is a cationic disodium salt, while the micronized dex in the present study is anionic (also available as an acetate salt). Based on electrical charge, the anionic form of dex would be expected to accumulate to a greater degree under the influence of the endocochlear potential, although a greater accumulation of the anionic form is certainly not apparent from our measurements. Alternatively, it is possible that the permeation properties of the anionic and cationic drug forms through the endolymphatic boundary may differ. In addition, the difference could have arisen from technical issues associated with fluid sampling. In the study by Parnes et al., large samples of perilymph were taken from the basal turn of the cochlea, a procedure which is known to cause dilution of the perilymph with cerebrospinal fluid, resulting in low measured perilymph concentrations [Plontke and Salt, 2003].

\section{Acknowledgments}

This work was supported by research grant DC01368 from NIDCD, NIH. The drug formulation used in this study was provided by Luis Dellamary of Otonomy, Inc.

\section{Disclosure Statement}

Dr. Salt is a member of the Scientific Advisory Board of Otonomy and may receive income based on equity holdings. This study was conducted independently and was not financially supported by Otonomy Inc. Drs. LeBel and Piu are current employees of Otonomy Inc.

\section{References}

Alles MJ, der Gaag MA, Stokroos RJ: Intratympanic steroid therapy for inner ear diseases, a review of the literature. Eur Arch Otorhinolaryngol 2006;263:791-797.

-Arnold W, Senn P, Hennig M, Michaelis C, Deingruber K, Scheler R, Steinhoff HJ, Riphagen F, Lamm K: Novel slow- and fasttype drug release round-window microimplants for local drug application to the cochlea: an experimental study in guinea pigs. Audiol Neurootol 2005;10:53-63.

- Bird PA, Begg EJ, Zhang M, Keast AT, Murray DP, Balkany TJ: Intratympanic versus intravenous delivery of methylprednisolone to cochlear perilymph. Otol Neurotol 2007;28: 1124-1130.

Borden RC, Saunders JE, Berryhill WE, Krempl GA, Thompson DM, Queimado L: Hyaluronic acid hydrogel sustains the delivery of dexamethasone across the round window membrane. Audiol Neurootol 2010;16:1-11.

-Chandrasekhar SS, Rubinstein RY, Kwartler, JA, Gatz M, Connelly PE, Huang E, Baredes S: Dexamethasone pharmacokinetics in the inner ear: comparison of route of administration and use of facilitating agents. Otolaryngol Head Neck Surg 2000;122:521-528.

Czock D, Keller F, Rasche FM, Häussler U: Pharmacokinetics and pharmacodynamics of systemically administered glucocorticoids. Clin Pharmacokinet 2005;44:61-98.
Endo T, Nakagawa T, Kita T, Iguchi F, Kim TS, Kopke RD, Hoffer ME, Wester D, O’Leary MJ, Tamura T, Iwai K, Tabata Y, Ito J: Novel strategy for treatment of inner ears using a biodegradable gel. Laryngoscope 2005;115:20162020.

Gouveris H, Selivanova O, Mann W: Intratympanic dexamethasone with hyaluronic acid in the treatment of idiopathic sudden sensorineural hearing loss after failure of intravenous steroid and vasoactive therapy. Eur Arch Otorhinolaryngol 2005;262:131-134.

- Hahn H, Kemmerer B, DiMauro A, Salt AN, Plontke S: Cochlear microdialysis for quantification of dexamethasone and fluorescein entry into scala tympani during round window administration. Hear Res 2006;212: 236-244.

Hentzer E: Ultrastructure of the middle ear mucosa. Acta Otolaryngol Suppl 1984;414:19-27.

Herr BD, Marzo SJ: Intratympanic steroid perfusion for refractory sudden sensorineural hearing loss. Otolaryngol Head Neck Surg 2005;132:527-531.

Hu A, Parnes LS: Intratympanic steroids for inner ear disorders: a review. Audiol Neurootol 2009; 14:373-382.

-James DP, Eastwood H, Richardson RT, O'Leary SJ: Effects of round window dexamethasone on residual hearing in a Guinea pig model of cochlear implantation. Audiol Neurootol 2008; 13:86-96

Kim SH, Kim KX, Raveendran NN, Wu T, Pondugula SR, Marcus DC: Regulation of ENaCmediated sodium transport by glucocorticoids in Reissner's membrane epithelium. Am J Physiol Cell Physiol 2009;296:C544C557. Jackson RL: Targeted topical steroid therapy in sudden sensorineural hearing loss. Otol Neurotol 2001;22:475-479.

Mikulec AA, Hartsock JJ, Salt AN: Permeability of the round window membrane is influenced by the composition of applied drug solutions and by common surgical procedures. Otol Neurotol 2008;29:1020-1026.

Mikulec AA, Plontke SK, Hartsock JJ, Salt AN: Entry of substances into perilymph through the bone of the otic capsule following intratympanic applications in guinea pigs: implications for local drug delivery in humans. Otol Neurotol 2009;30:131-138.

- Mynatt R, Hale SA, Gill RM, Plontke SKR, Salt AN: Demonstration of a longitudinal concentration gradient along scala tympani by sequential sampling of perilymph from the cochlear apex. J Assoc Res Otolaryngol 2006; 7:182-193.

Paulson DP, Abuzeid W, Jiang H, Oe T, O’Malley BW, Li D: A novel controlled local drug delivery system for inner ear disease. Laryngoscope 2008;118:706-711.

- Parnes LS, Sun AH, Freeman DJ: Corticosteroid pharmacokinetics in the inner ear fluids: an animal study followed by clinical application. Laryngoscope 1999;109:1-17.

Plontke SK, Salt AN: Quantitative interpretation of corticosteroid pharmacokinetics in inner fluids using computer simulations. Hear Res 2003; $182: 34-42$. 
Plontke SK, Salt AN: Simulation of application strategies for local drug delivery to the inner ear. ORL J Otorhinolaryngol Relat Spec 2006;68:386-392.

- Plontke SK, Biegner T, Kammerer B, Delabar U, Salt AN: Dexamethasone concentration gradients along scala tympani after application to the round window membrane. Otol Neurotol 2008a;29:401-406.

Plontke SK, Mikulec AA, Salt AN: Rapid clearance of methylprednisolone after intratympanic application in humans. Otol Neurotol 2008b;29:732-733.

Plontke SK, Löwenheim H, Mertens J, Engel C, Meisner C, Weidner A, Zimmermann R, Preyer S, Koitschev A, Zenner HP: Randomized, double blind, placebo controlled trial on the safety and efficacy of continuous intratympanic dexamethasone delivered via a round window catheter for severe to profound sudden idiopathic sensorineural hearing loss after failure of systemic therapy. Laryngoscope 2009;119:359-369.
Pondugula SR, Raveendran NN, Ergonul Z, Deng Y, Chen J, Sanneman JD, Palmer LG, Marcus DC: Glucocorticoid regulation of genes in the amiloride-sensitive sodium transport pathway by semicircular canal duct epithelium of neonatal rat. Physiol Genomics 2006;24:114-123.

Salt AN, Gill RM, Plontke SK: Dependence of hearing changes on the dose of intratympanically applied gentamicin: a metaanalysis using mathematical simulations of clinical drug delivery protocols. Laryngoscope 2008; 118:1793-1800.

Salt AN, Plontke SK: Principles of local drug delivery to the inner ear. Audiol Neurootol 2009;14:350-360.
Schuknecht HF: Pathology of the Ear. Philadelphia, Lea and Febiger, 1993.

-Selivanova OA, Gouveris H, Victor A, Amedee RG, Mann W: Intratympanic dexamethasone and hyaluronic acid in patients with low-frequency and Ménière's-associated sudden sensorineural hearing loss. Otol Neurotol 2005;26:890-895.

Tamura T, Kita T, Nakagawa T, Endo T, Kim TS, Ishihara T, Mizushima Y, Higaki M, Ito J: Drug delivery to the cochlea using PLGA nanoparticles. Laryngoscope 2005; 115: 2000-2005.

Van Wijck F, Staecker H, Lefebvre PP: Topical steroid therapy using the Silverstein Microwick in sudden sensorineural hearing loss after failure of conventional treatment. Acta Otolaryngol 2007;127:1012-1017.

-Wang X, Dellamary L, Fernandez R, et al: Dosedependent sustained release of dexamethasone in inner ear cochlear fluids using a novel local delivery approach. Audiol Neurootol 2009; 14:393-401. 